\title{
Extracellular vesicles from stem and progenitor cells for cell-free regenerative therapy
}

Article

Accepted Version

Haque, N., Widera, D. ORCID: https://orcid.org/0000-00031686-130X, Govindasamy, V., Soesilawati, P. and Kasim, N. H. A. (2022) Extracellular vesicles from stem and progenitor cells for cell-free regenerative therapy. Current Molecular Medicine, 22 (2). pp. 120-131. ISSN 1566-5240 doi:

https://doi.org/10.2174/1566524021666210125114828 Available at https://centaur.reading.ac.uk/95732/

It is advisable to refer to the publisher's version if you intend to cite from the work. See Guidance on citing.

To link to this article DOI:

http://dx.doi.org/10.2174/1566524021666210125114828

Publisher: Bentham Science Publishers

All outputs in CentAUR are protected by Intellectual Property Rights law, including copyright law. Copyright and IPR is retained by the creators or other copyright holders. Terms and conditions for use of this material are defined in the End User Agreement. 


\section{CentAUR}

Central Archive at the University of Reading

Reading's research outputs online 


\title{
Extracellular Vesicles from Stem and Progenitor Cells for Cell-Free Regenerative Therapy
}

Nazmul Haque ${ }^{* a}$, Darius Widera ${ }^{\mathrm{b}}$, Vijayendran Govindasamy ${ }^{\mathrm{c}}$, Pratiwi Soesilawati ${ }^{\mathrm{d}}$, Noor Hayaty Abu Kasim $^{*}$ de

${ }^{a}$ Department of Oral Biology and Biomedical Sciences, Faculty of Dentistry, MAHSA University, Selangor, Malaysia.

${ }^{b}$ Stem Cell Biology and Regenerative Medicine Group, School of Pharmacy, University of Reading, Reading, UK

${ }^{c}$ Cryocord Malaysia Sdn Bhd, Cyberjaya, Selangor, Malaysia

$\underline{\underline{d}}$ Faculty of Dental Medicine, Universitas Airlangga, Surabaya, Indonesia.

${ }^{e}$ Faculty of Dentistry, Universiti Kebaangsan Malaysia, Kuala Lumpur, Malaysia.

\begin{abstract}
Cell-based regenerative therapies involving stem or progenitor cells are considered as possible therapeutic modalities to treat non-communicable and degenerative diseases. Recently, regenerative outcomes of cell-based therapies have been linked to paracrine factors and extracellular vesicles [EVs] released by the transplanted cells rather than the transplanted cells themselves. EVs contain a cargo that includes microRNAs [miRNAs], mRNAs, as well as proteins. Their role in mediating intercellular communication has been acknowledged in several studies. However, the regenerative potential of the miRNAs, mRNAs, and proteins that are present in EVs is a matter of ongoing scientific debate. In this review, we discuss EVs as an alternative to stem cell-based therapy to treat some of the non-communicable and degenerative diseases. Moreover, we also propose that pre-treatment of the cells could help to produce EVs enriched with particular miRNAs, mRNAs, and/or proteins that could support the successful regeneration of a targeted organ.
\end{abstract}

Keywords: exosomes; mesenchymal stem cells; microvesicles; miRNA; mRNA; paracrine factor. 


\section{INTRODUCTION}

In the last four decades, the average human life expectancy at birth has increased by about 10 years [1-3]. However, simultaneously, a 9 years gap between the total life expectancy and healthy life expectancy has also been reported [1]. This gap between life expectancy and healthy life expectancy indicates that a large number of people around the world are living with medical conditions. Noncommunicable and age-related degenerative diseases are considered the major contributors to these morbid conditions [1-3]. There are several symptomatic treatments available to treat non-communicable or degenerative diseases. However, till now there is no effective therapeutic modality that could help to regenerate affected organs. Often, organ transplantation is the only option, whereas scarcity in donor organs and immunologically mediated graft loss following transplantation are the major drawbacks [4, 5].

Several in vitro, in vivo, and clinical studies have been conducted with a large variety of stem cell types including induced pluripotent stem cells [iPSCs], mesenchymal stem cells [MSCs], hematopoietic stem cells [HSCs], blast cells, and progenitor cells. Their promising regenerative outcomes are considered as hope to resolve the issue of organ donor scarcity in the near future [6-15].

\footnotetext{
*Address correspondence to this author at the Noor Hayaty Abu Kasim, Faculty of Dentistry, Universiti Kebangsaan Malaysia, 50300 Kuala Lumpur, MALAYSIA. E-mail: nhayaty@ukm.edu.my
}

Nazmul Haque, Department of Oral Biology and Biomedical Sciences, Faculty of Dentistry, MAHSA University, 42610 Selangor, MALAYSIA.

E-mail: tanna.bge@gmail.com; nazmul@mahsa.edu.my
However, several studies have suggested that the regenerative outcomes of cell therapy, especially of MSCs based regenerative therapy, are largely mediated by paracrine factors rather than by engraftment and differentiation of the transplanted cells [16-18]. This emphasizes the regenerative potential of stem cell-derived paracrine factors and extracellular vesicles [EVs]. This opened a new opportunity in the field of regenerative medicine and led to multiple studies exploring the potential of stem cell secretome and EVs as cell-free regenerative therapeutics.

Release of EVs by reticulocytes into the extracellular space was observed and reported in 1980s [19, 20]. Until 1996, before exploring the role of EVs in stimulating an adaptive immune response [21], they were considered as cell debris without biological relevance [22]. After that discovery, EVs received considerable attention due to their ability to mediate intercellular communication.

Based on their biogenesis, EVs can be divided into exosomes, microvesicles, and apoptotic bodies [Figure 1] [22]. Exosomes are released by cells upon fusion of multivesicular endosomes with the plasma membrane [23] and range in size from $30-120 \mathrm{~nm}$ in diameter [24]. Microvesicles originate from the cell surface by direct outward budding and fission of the plasma membrane [24]. They are more heterogeneous compared to exosomes and range from 50-1000 $\mathrm{nm}$ in diameter [25]. Among different types of EVs, apoptotic bodies that are generated through a process known as apoptotic cell disassembly, represent the largest EVs with an average diameter of 1-5 $\mu \mathrm{m}$ [26].

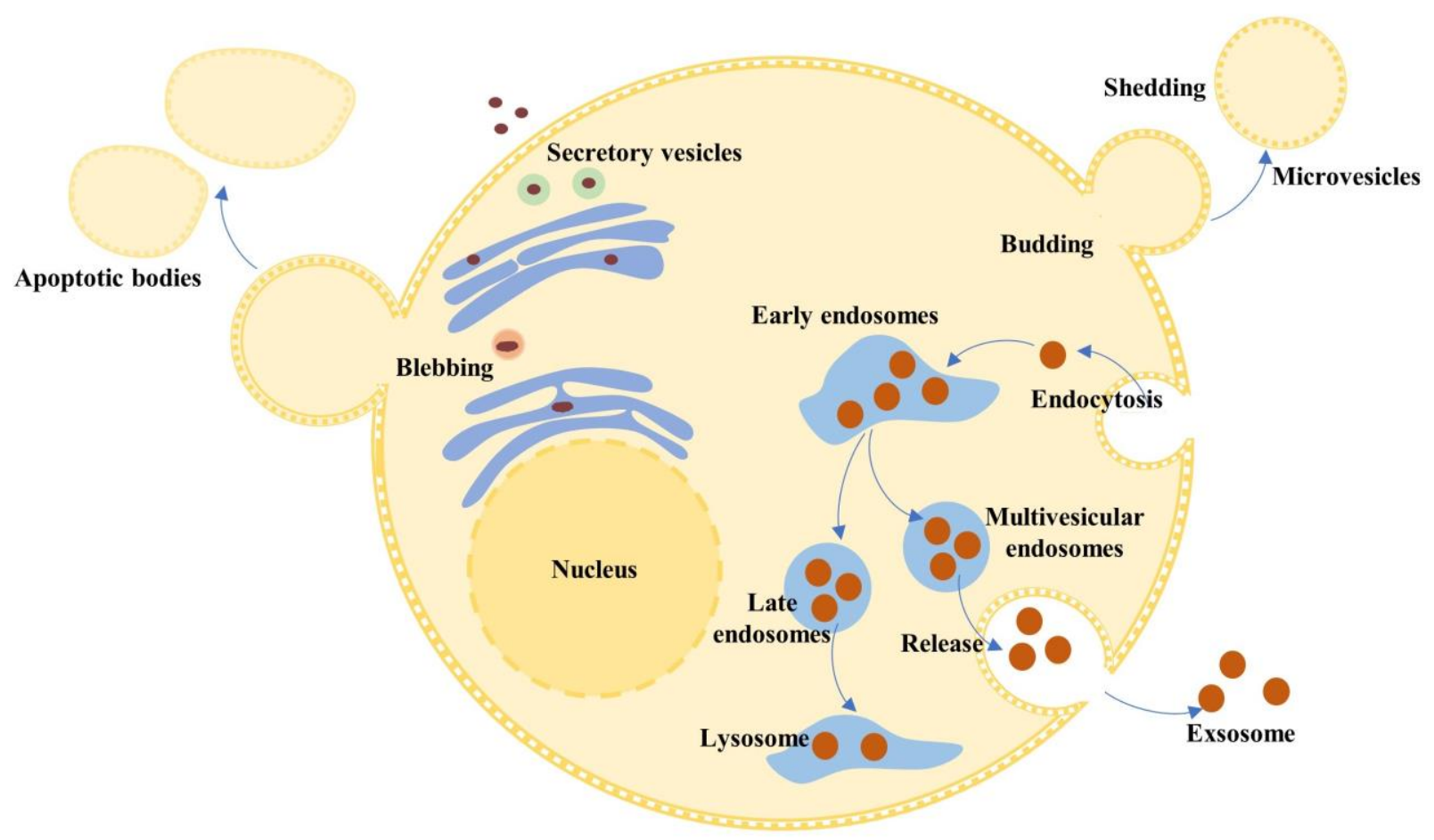

Figure 1 Different types of extracellular vesicles (EVs) and their biogenesis. 
EVs contain a cargo that includes microRNAs [miRNAs], mRNAs, and proteins which play a vital role in maintaining intercellular communication [26, 27]. In recent years, the regenerative potential of EVs [especially exosomes and microvesicles] has also received considerable attention. In this review, the regenerative potential and future prospects of EVs as cell-free therapeutics will be discussed.

\section{POTENTIAL USES OF EVS IN REGENERATIVE THERAPY}

Cell-cell communication through EVs is a bidirectional process [28]. Usually, the biogenesis and the content of EVs are closely linked to the cross-talk between cells and their niche. Cells of origin and the physiologic or pathologic condition of the microenvironment regulate the content of the EVs and subsequently their biological functions [29]. Following secretion, EVs carry signaling molecules [miRNAs, mRNA, and proteins] to adjacent or distant cells and play an important role in maintaining cellcell communication [30]. The EVs originating from a particular cell type can regulate the phenotype and fate of the recipient cells by transferring cell surface receptors, genetic material, and/or transcriptional regulators [31, 32]. The regenerative potential of EVs from different cell types has been studied in vitro and in vivo. In the following, we will discuss the potential of EVs in treating selected pathological conditions including cardiac, liver, and neuronal degeneration [Figure 2].

\subsection{Cardiac Regeneration}

In the last few decades, myocardial infarction has been recognized as one of the major causes of mortality and morbidity worldwide. It also has a significant impact on the socio-economic status and quality of life of the survivors [33]. Cell-cell communication or cross-talk between different cell types including cardiac progenitor cells, cardiomyocytes, endothelial cells, and fibroblasts play vital roles in cardiac regeneration and protection of the ischemic myocardium. To maintain homeostasis within heart tissue, cardiomyocytes send messages to cardiac progenitor cells, endothelial cells, and fibroblasts while receiving reciprocal signals from these cell types [34].

Endothelial progenitor cells [EPCs] and MSCs have the potential to protect the ischemic myocardium [35]. Recently, it has been established that EVs are the key component of cell-cell communication and the cardiac regenerative outcomes of the cell-based therapy are due to cell-secreted paracrine factors embedded within EVs [33, $36,37]$. In this context, the protective effects of hiPSC- and cardiomyocyte-derived EVs on ischemic myocardium have been reported [34, 38]. However, the molecular mechanisms behind the EV-mediated myocardial protection are still illusive.

Previously, it has been hypothesized that proteomic deficiencies during ischemia-reperfusion injury may be linked to the loss of adenosine triphosphate [ATP]/ nicotinamide adenine dinucleotide hydrogen [NADH], resulting in increased oxidative stress and cell death [39, 40]. Increased ATP and NADH levels, decreased oxidative stress, increased phosphorylated protein kinase B [p-Akt] phosphorylated glycogen synthase kinase-3 [p-GSK-3] beta, and reduced C-Jun N-terminal kinase [p-c-JNK] within one hour after reperfusion in ischemic mice hearts treated with MSC-derived EVs [MSC-EVs] have been reported. Furthermore, $24 \mathrm{~h}$ post-reperfusion, a significant reduction in local and systemic inflammation, and a $45 \%$ reduction in infarct size were observed in MSC-EVs treated animals. Higher contractility and relaxation have also been observed during a 28 days follow-up. However, the effect was confined to the intact EVs only [40]. In another study, it has been found that cluster of differentiation [CD] 81, CD9, and Alix markers containing purified EVs reduced infarct size in the mouse of myocardial ischemia-reperfusion injury model [37].

Several in vitro and in vivo studies have linked the presence of miRNAs in EVs to the protection of cardiomyocytes, and induction of angiogenesis [34, 41]. In an in vivo study, intravenously injected apoptotic human umbilical vein endothelial cell [HUVEC]-EVs induced a reduction of inflammatory atherosclerotic plaques [42]. This regenerative outcome was further linked to the presence of miR-126 in the EV fraction that induced the cardiac protection by increasing the expression of stromal cellderived factor 1 [SDF-1] in recipient cells [42]. In vitro protection of cardiac progenitor cells [CPCs] under ischemic condition by miR-126 and miR-210 enriched EVs have been acknowledged in another study [43]. Similar observations have been reported for the EVs containing miR-210, miR132, and miR-146a released by cardiosphere-derived cells and CPCs [41, 44]. Recently, multilayered composite membranes of biomaterials have been reported to increase the secretion of EVs from EPCs in which a higher amount of miR-126 [45]. The results of this study suggest that the culture of stem cells on the multilayered composite membrane of biomaterials could help to produce EVs with higher angiogenic potential.

In addition to miRNAs, EV cargo also contains multiple proteins. Under physiological conditions, [SDF-1]/ C-X-C motif chemokine receptor [CXCR4] signaling has been reported to be involved in enhanced endothelial sprouting from human embryoid bodies [46]. This result signifies the role of CXCR4 in regulating initial vessel formation which is highly important for cardiac regeneration. In another study, EVs from MSCs transduced with lentiviral CXCR4 have been found to promote functional cardiac restoration [increased angiogenesis, reduced infarct size, and improved cardiac remodeling] in vivo via upregulating the expression of insulin- like growth factor 1 alpha [IGF-1 $\alpha$ ] alpha, pAkt, vascular endothelial growth factor [VEGF], as well as by downregulating the levels of active caspase 3 in cardiomyocytes [47]. Further studies are needed to reveal and specify the role of EVs from different sources or the components of EVs in cardiac regeneration.

\subsection{Liver Regeneration}

Hepatic fibrosis is a process that results from imbalanced accumulation and degradation of the extracellular matrix [48]. It is triggered by chronic liver injuries. If untreated, it may cause life-threatening liver cirrhosis and hepatocellular carcinomas. Chronic inflammation of hepatic cells is frequently caused by viral infections, alcoholic liver disease, non-alcoholic fatty liver disease, and autoimmune attack. Liver cirrhosis an endstage sequela of fibrosis is one of the major causes of morbidity and mortality worldwide. At present, there is no pharmaceutical option for treating hepatic fibrosis, and liver transplantation is considered the only curative treatment for end-stage cirrhosis [5]. However, scarcity in the donor organs even in the developed countries as well 
as low survival rates of grafts following liver transplantation are the major limitations of this treatment modality. These obstacles intensified the need of new medical therapies to attenuate or reverse hepatic fibrosis.

Several in vitro and in vivo studies have demonstrated the hepatic regenerative potential of EVs from different cell types. In vitro inhibition of growth and survival of HepG2 hepatoma and primary hepatocellular carcinoma cells in the presence of human adult liver stem cells [HLSC]-EVs have been reported earlier [49]. Furthermore, intra-tumor administration of HLSC-EVs have shown regression of ectopic tumors in severe combined immunodeficiency [SCID] mice [49]. These hepatoprotective effects of HLSC-EVs were linked to a horizontal transfer of miRNAs from EVs to the target cells [49]. In another study, EVs from bone marrow [BM]MSCs also induced apoptosis of HepG2 and Kaposi's cells as well as necrosis in Skov-3 in vitro in addition to an inhibition of progression of established tumors in vivo [50].

EVs from different cell sources have also been shown to have the potential to minimize ischemia-reperfusion and toxin-induced hepatic injury. It was reported that EVs from human induced pluripotent stem cell [iPSCs] derived MSCs reduced the levels of the hepatic injury markers aspartate aminotransferase [AST] and alanine aminotransferase [ALT], multiple pro-inflammatory proteins including tumor necrosis factor [TNF]-alpha, interleukin [IL]-6, high mobility group box 1 [HMGB1] as well as the apoptotic markers caspase-3 and bax [51]. Moreover, EVs increased the expression levels of oxidative markers, such as glutathione [GSH], glutathione peroxidase [GSH-Px], and superoxide dismutase [SOD] [51]. In a toxicity-induced [n-acetyl-p aminophenol or hydrogen peroxide] in vitro injury model, EVs from hepatic stellate cells [HSC] LX-2 and HST-T6 also improved the viability, and inhibited the apoptosis, and reduced cleaved caspase- 3 expression as well as the leakage of lactate dehydrogenase [LDH], ALT, and AST from hepatocytes in a dose-dependent manner [52].

Efficacy of EVs from human foetal tissue-derived MSCs and umbilical cord [UC]-MSCs in treating hepatic fibrosis has also been reported earlier [53, 54]. However, the role of $\mathrm{MSC}$ - derived $\mathrm{EVs}$ in regulating molecular pathways involved in hepatic fibrosis remains to be explored. Transdifferentiation or activation of quiescent hepatic stellate cells [qHSCs] into proliferative and fibrogenic myofibroblast like activated HSCs [aHSCs] has been identified as the key cause of liver fibrosis $[55,56]$. EVs from MSCs are rich in paracrine factors and their role in immunomodulation has been widely acknowledged [57]. EVs from MSCs have been shown to possess the potential to regulate the activation of HSCs and to reduce hepatic fibrosis $[53,54]$. It has been hypothesized that the presence of antifibrogenic miRNAs [miR-15a, miR-15b, miR-16, miR-29a, miR-195] in the EVs might have the potential to regulate the expression of genes involved in the HSCs activation such as collagen 1 [Colla1], actin alpha 2 [Acta2], transforming growth factor beta receptor 1 [Tgfbr1], and tissue inhibitors of metalloproteinases [Timp1] [56, 58, 59]. Furthermore, expression of the targeted miRNAs in the EVs from MSCs could be induced and used as a potential therapeutic modality.

On the basis of all the research outcomes, it can be postulated that by inhibiting activation of hepatic stellate cells, EVs could show its hepatoprotective effect. Meanwhile, by minimizing oxidative stress, modulating immune response and increasing hepatocyte proliferation EVs could contribute to liver regeneration.

\subsection{Neuronal Regeneration}

Acute ischemic stroke and neurodegenerative disorders such as Alzheimer's disease and Parkinson's disease are among the leading causes of death and disability in the world. The potential of cell-based regenerative therapy has been being studied in the last two decades. In recent years, the neuroregenerative potential of secretomes and EVs from different stem and progenitors has been explored [6062].

Lower concentration of EVs [from murine adiposederived [AD]-MSC have been found to protect human neuroblastoma SH-SY5Y cells and primary murine hippocampal neurons from chemically induced oxidative stress [61]. Similarly, increased remyelination and activated nestin-positive oligodendroglial precursors in the cerebral slice from C57BL/6 mice pups if exposed to a lower concentration of AD-MSC-EVs [61]. The authors concluded that AD-MSC-EVs could be beneficial in preventing neuro-inflammation and treating neurodegenerative disorders [61]. EVs from dental pulp stem cells [SHED] cultured on the laminin-coated threedimensional alginate micro-carriers have been found to prevent apoptosis in dopaminergic neurons in the presence of the toxic dopamine derivative 6-hydroxy-dopamine [6OHDA] [62].

In another study, an increased expression of miR-133b in the ipsilateral hemisphere of rats subjected to middle cerebral artery occlusion [MCAo] was seen when treated with MSCs. In the presence of ipsilateral ischemic tissue extracts from rats subjected to MCAo also induced the expression of miR-133b in MSCs and their exosomes in vitro. Furthermore, treatment with exosome-enriched fractions yielded from the MSCs induced expression of miR-133b in primary cultured neurons and astrocytes. The knock-down of miR-133b in MSCs confirmed that the increased miR-133b level in astrocytes is attributed to their transfer from MSCs. Exosome-enriched fractions from MSCs exposed to post-MCAo brain extracts also significantly increased the neurite branch number and total neurite length. This study revealed that MSCs communicate with brain parenchymal cells via exosomes and may regulate neurite outgrowth by transfer of miR$133 \mathrm{~b}$ to neural cells [63]. Knock-in and knock-down of miR-133b further confirmed that neurological recovery following a stroke in rats was mediated by the transfer of miR-133b containing exosomes from MSCs [64].

Spinal cord injury $[\mathrm{SCI}]$ is a condition causing morbidity due to irreversible motor dysfunction and death as well. Several studies have reported the potential of EVs derived from different sources in improving motor function following SCI. Rong et al. [2019] reported the potential of neural stem cells derived EVs in reducing the extent of SCI and improving functional recovery of motor neurons in rats. This was further linked to the induced expression of autophagy proteins namely LC3B and beclin-1; boosted autophagosome formation; and reduced expression of apoptotic regulator Bax, apoptosis effector cleaved caspase-3, and pro-inflammatory paracrine factors TNF- $\alpha$, IL-1 $\beta$, and IL-6 [65]. BMSC-EVs have also been reported to improve motor function in the rat by reducing 
neuronal death and inducing neuronal survival and regeneration. Furthermore, BMSC-EVs improved blood spinal cord barrier integrity by downregulating $\mathrm{NF}-\kappa \mathrm{B}$ signaling in pericytes [66]. In another in vivo study, UCMSC-EVs were found to improve SCI by reducing proinflammatory cytokines expression and scar formation [67]. Notably, in a recent study, motor function improvement in SCI rats was linked to the presence of miRNA-29b in the exosomes [68]. Improved hind-limb movements and bladder control in chronic SCI dogs following multiple intravenous injections of BMMSC secretome further denote the neuro-regenerative potential of EVs [69].

Crossing the blood-brain barrier of therapeutic agents is the major challenge in treating neurodegenerative diseases. In this context, it has been demonstrated that EVs have the potential to cross the blood-brain barrier [70]. All these studies encourage further development of engineered exosomes as drug delivery vesicles or tracking tools for treating or monitoring neurological diseases.

\subsection{Wound Healing}

The prevalence of acute and chronic wounds is continuously increasing and being a major cause of morbidity. Acute wounds are caused by thermal injury, trauma, excision of extensive skin cancer, deep fungal and bacterial infections, vasculitis, scleroderma, pemphigus, toxic epidermal necrolysis, etc. Chronic wounds include diabetic ulcers, bedsore, arterial [ischemic] ulcers, and venous ulcers. These diseases increase the health care cost and thus the economic burden. Importantly, conventional treatments for these conditions are often ineffective [71]. Several in vitro and in vivo studies using EVs from different sources have been conducted to explore their potential in treating acute and chronic wounds.

New blood vessel formation is essential in wound healing. Crosstalk between endothelial cells, paracrine factors, and extracellular matrix [ECM] proteins play a vital role in the process of regeneration. Interestingly, dose-dependent uptake of BM-MSC-EVs by endothelial cells and an increased tube formation was reported in vitro. This was linked to activation of important wound healing signaling pathways [Akt, extracellular signal-regulated kinase [ERK], and signal transducer and activator of transcription [STAT3]] and induced expression of a number of paracrine factors [ hepatocyte growth factor [HGF], insulin-like growth factor [IGF1], nerve growth factor [NGF], and SDF1] [72]. The presence of mRNA in the EPC-EVs has also been reported to induce angiogenesis in endothelial cells by regulating the phosphatidylinositol 3-kinase [PI3K]/Akt signaling pathway [73]. Among the EPCs subpopulations, EPCs with low activity of aldehyde dehydrogenase [Alde-Low EPCs] have been found to be biologically more active in repairing ischemic tissues [74]. Later it was reported that Alde-Low EPCs express a higher amount of hypoxia inhibition factor [HIF]-2 and its target gene CXCR4 [75]. Improved ability to repair an ischemic skin flap along with the significantly higher expression of CXCR4 and its ligand SDF1 in Alde-High EPCs in the presence of EVs from Alde-Low EPCs subpopulation was also reported [75]. These studies signify the role of EVs from Alde-Low EPCs in wound healing by regulating the cellular migration and blood vessel formation.
Preventing myofibroblast formation and accumulation is another major challenge in scar-free wound healing. EVs from umbilical cord-derived MSCs enriched in specific miRNAs such as miR-21, miR-23a, miR-125b, and miR-145 have been reported to play key roles in suppressing myofibroblast formation by inhibiting the transforming growth factor-beta 2 [TGF- $\beta 2$ ]/SMAD2 pathway [76]. Significantly accelerated reepithelialization, and increased expression of CK19, proliferating cell nuclear antigen [PCNA], collagen I [compared to collagen III] in deep second-degree burned rat skin treated with hUC-MSC-EVs was also shown. Induced wound re-epithelialization and reduced heat stress-induced apoptosis of cells in the hUC-MSC-EVs treated rat were further connected to the activation of Wnt/-catenin and Akt signaling pathways respectively [77]. Another study was conducted to reveal the potential of MSC-EVs in controlling overcrowding and dysplasia following stem cells expansion after a regenerative response. The researchers have reported that hUC-MSCEVs contain 14-3-3 $\zeta$. Under high cell density conditions, $14-3-3 \zeta$ regulates the binding of YAP and phosphorylated large tumor suppressor kinase [LATS] by forming a complex to promote the phosphorylation of yes-associated protein [YAP] followed by inhibition of Wnt4 /-catenin signaling cutaneous regeneration [78]. Outcomes of these studies indicate that that hUC-MSC-EVs not only induce wound healing by activating $\mathrm{Wnt} /$-catenin signaling but also regulate YAP to control cutaneous regeneration.

\subsection{Bone Regeneration}

The incidence of bone disorders has been increasing worldwide underlining the necessity of regenerative therapies for bone tissue. Like in other tissues, cell fate and bone regeneration are also influenced by EVs secreted by cells residing within the injured tissue. Lineage-specific expression of miRNA in ESCs in the presence of preosteoblast-EVs has been reported earlier [79]. Studies have also shown that EVs collected from both BM-MSCs regular culture medium and osteogenic differentiation medium have the potential to induce osteogenic differentiation of naive BM-MSCs [80, 81]. However, the lineage-specific differentiation of naive BM-MSCs in vitro and in vivo was higher in the presence of EVs isolated from osteogenic differentiation media [81]. EVs collected from both cell culture conditions have revealed a binding capacity to matrix proteins such as type I collagen and fibronectin [81]. These results suggest the potential future use of EVs to pretreat autologous cell populations prior to transplantation to improve the bone regenerative outcomes [81].

In another study, fracture healing in CD9[-/-] mice was accelerated by BM-MSCs derived EVs, despite having the lower levels of the bone repair-related cytokines such as monocyte chemotactic protein-1 [MCP-1], MCP-3, and SDF1 compared to the levels in BM-MSCs condition medium and EVs free condition medium [82]. This result highlights the role of miRNAs in accelerating the bone repairing capability of EVs.

\subsection{Graft versus Host Disease}

Several studies reported MSCs as being immune evasive rather than immune privileged [83, 84]. Although there are controversies regarding the immunomodulatory properties of MSCs, several studies have acknowledged their potential in preventing life-threatening graft versus 
host disease [GVHD] [85-87]. Similar to the regenerative and immunomodulatory potentials of MSCs themselves, immunomodulatory properties of MSC-derived EVs have also received remarkable attention [88-90].
IFN- $\gamma$ from patient-derived natural killer $[\mathrm{NK}]$ cells in the presence of MSC-EVs have also been reported in a clinical study [93]. Notably, the immunomodulatory properties of EVs are linked to the internalization of the EVs by the

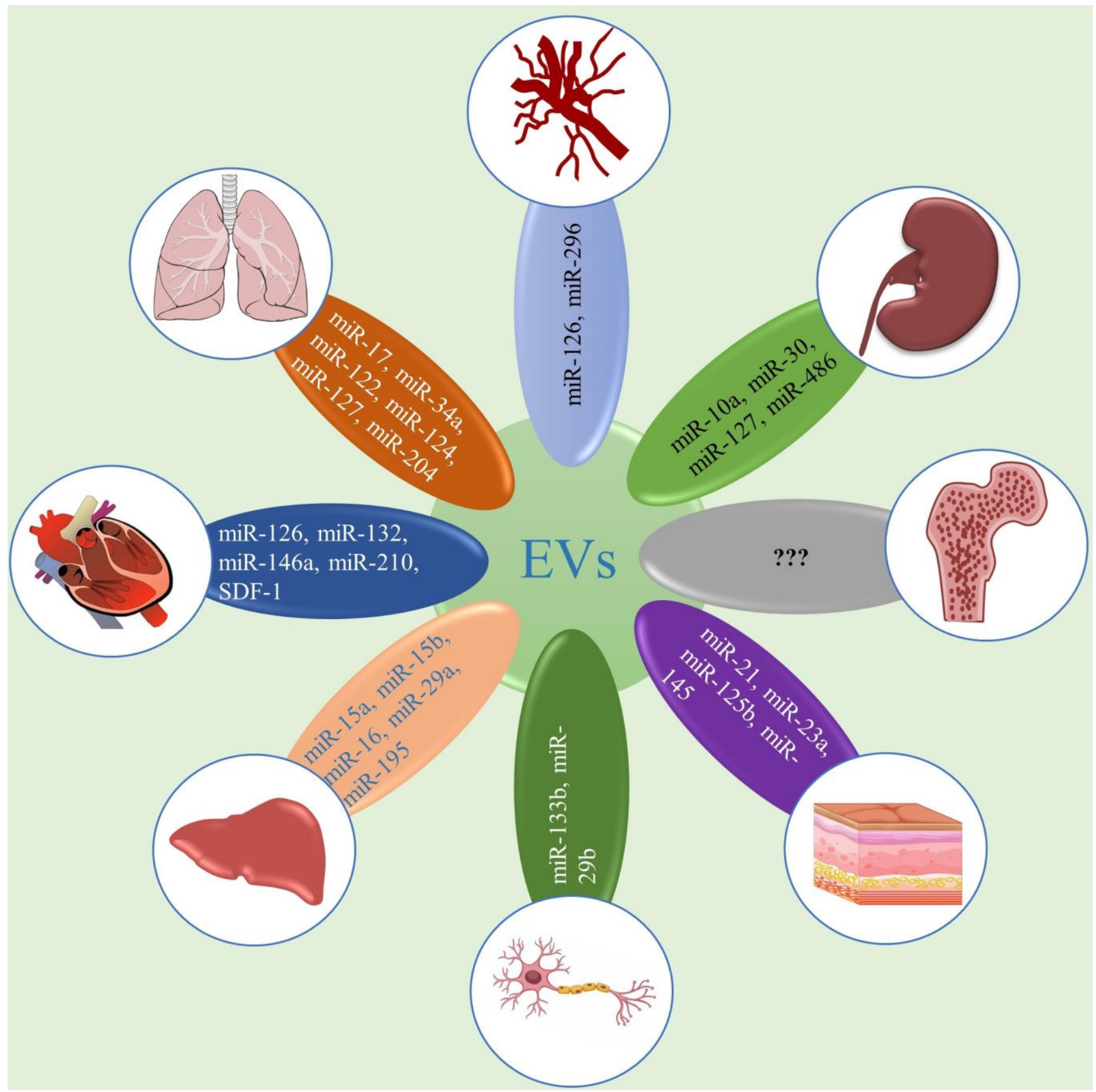

Figure 2 Components of extracellular vesicles for tissue or organ-specific regenerative therapy.

In an in vivo study, the infusion of hUC-MSC-EVs into mice following allogeneic hematopoietic stem cell therapy [HSCT] showed a prophylactic effect and significantly reduced the mortality of the recipient mice. These EVs significantly reduced the numbers of cytotoxic/suppressor $\mathrm{T}$ cells and the expression of paracrine factors [IL-2, TNF$\alpha$, and interferon [IFN]- $\gamma$ ] in the serum. Simultaneously, it increased the ratio between $\mathrm{CD} 3{ }^{+} \mathrm{CD} 4^{+}$and $\mathrm{CD} 3{ }^{+} \mathrm{CD} 8^{+} \mathrm{T}$ cells and elevated the expression levels of IL-10. [91]. In another study, BM-MSC-EVs have been shown to induce apoptosis of $\mathrm{CD}^{+}$cells including the $\mathrm{CD}^{+}$subpopulation. Furthermore, it increased proliferation and apoptosis of Tregs, the Treg/Teff ratio, and the levels of the immunosuppressive cytokine IL-10 in cell culture supernatants [92]. Reduced expression of IL-1 $\beta$, TNF- $\alpha$, and IFN- $\gamma$ from the patient-derived PBMC or TNF- $\alpha$ and cells [94].

There are controversies on the reduction of GVHD by MSC-EVs [95]. However, the positive outcomes of the most of the studies necessitate to study the compositions [proteins, mRNA and miRNA] and immunomodulatory properties of EVs to find out the key components involved in the innate and adaptive immune regulation and to translate their uses in preventing GVHD [87, 96].

\subsection{Kidney Regeneration}

The role of MSCs in preventing acute kidney injury by ameliorating renal dysfunction and repairing tubular damage has been acknowledged in several studies [97, 98]. However, the regenerative outcomes were facilitated by the paracrine effects of MSCs rather than their homing and 
differentiation to target cells [99]. In 2013, Tomasoni et al. reported that BM-MSC-EVs horizontally transfer IGF-1R mRNA to the tubular cells which enhanced tubular cell sensitivity to locally produced IGF-1 and prevent cisplatininduced damage of proximal tubular cells in vitro [100]. The role of hWJ-MSC-EVs in ameliorating ischemia/reperfusion- induced acute renal injury by inhibiting mitochondrial fission through delivering and restoring miR-30 expression in renal tissue has also been reported [101]. In another study, MSCs engineered with miRNAs [miR-10a, miR-127, and miR-296] maintained their pro-regenerative effects macrophage-mediated inflammation following acute ischemia/reperfusioninduced renal injury is one of the major cause of renal damage [102, 103]. Recently, CCR2 positive MSC-EVs prevented ischemia/reperfusion-induced renal injury by suppressing the functions of $\mathrm{C}-\mathrm{C}$ motif chemokine ligand [CCL2] that usually recruit or activate macrophage [104]. Thus, EVs from different cell sources could be developed as a therapeutic tool to treat kidney injuries.

\subsection{Human Islet Vascularization}

Pancreatic islet transplantation is considered as one of the treatment modalities in type 1 diabetes patients whose blood glucose level is difficult to control [105]. However, poor graft vascularization limits the functional benefits of islet transplantation. Cantaluppi et al. [2012] have shown enhanced human islet vascularization in response to EPCsEVs in vitro and in vivo. The authors have shown that following internalization of EPC-EVs by islet endothelium and beta-cells, insulin secretion, survival, and revascularization of transplanted islets in SCID mice was induced. EPC-EVs induced in vitro proliferation, migration, resistance to apoptosis, and neovascularization of islet endothelial cells. The authors have further linked the presence of miR-126 and miR-296 in EPC-EVs to an induced revascularization and insulin secretion potential of EPC-EVs in islet endothelial cells. Microarray analysis of islet endothelium further confirmed EPC-EVs mediated upregulation of mRNAs that are involved in endothelial proliferation, differentiation, and angiogenesis. Activation of P13K-Akt and eNOS signaling pathways which are vital for vascularization was also reported in EPC-EVs treated islet endothelium [106].

\subsection{Lung Repair and Regeneration}

Studies also reported the influence of lung derived EVs on the modification of fate and function of marrow cells $[107,108]$. These results suggest the use of lung cellderived EVs for priming the stem or progenitor cells prior to transplantation within a regenerative therapy. Furthermore, MSC-EV containing miR-34a, miR-122, miR-124, and miR-127 reported to improve the pulmonary hypertension in mice [109]. The potential of human MSCEVs in protecting the lung from E. coli endotoxin-induced acute lung injury in mice by expressing KGF mRNA in the injured alveolus has also been reported earlier [110].

\section{LIMITATIONS OF USING EVS IN REGENERATIVE THERAPY}

Within regenerative medicine, stem cells are being considered as a promising tool to treat incurable and difficult to treat diseases and conditions. However, in recent years, EVs have received substantial attention as potential cell-free surrogates to stem cell transplantation. EVs contain mainly miRNAs, mRNAs, and proteins that regulate bi- directional cell-cell communication. EVs can be used directly to treat any diseases or to precondition stem cells prior to transplantation to improve their regenerative or lineage-specific differentiation potential. Despite having regenerative potential, the composition of EVs varies vastly which limits its successful and replicable therapeutic use. Moreover, bio-distribution of endo- and exo-genously administered EVs have not been studied extensively [111]. Another concern is related to the use of xenogeneic serum and feeder cells in the production of EVs. It has been reported that hESCs maintained with xenogeneic feeder layer cells secrete a higher amount of particular proteins [annexin II, beta-actin, guanine nucleotide-binding protein, enolase 1, and cofilin] secrete EVs with a cargo involved in cellular metastasis and immortality which is usually present in invasive cancers. and [112]. Furthermore, like stem cells, xenogeneic serum, and feeder cells could contaminate the EVs with xeno-antigens that could affect their regenerative potential [112]. Finally, the safety of EVs, delivery mechanisms, potency of the EVs, and regulatory issues [manufacturing, standardizing, and up-scaling] need to be extensively studied for their successful use in regenerative medicine.

\section{CONCLUSION}

The use of EVs as cell-free therapeutics will help to minimize a lot of limitations related to the cell-based therapy. By analyzing the results from in vitro and in vivo studies on EVs, we attempted to categorize them on the basis of their contents and/or sources while considering their targeted regenerative potential. This categorization and enriching it with updated research outcomes will be very effective in selecting EVs to treat a particular tissue, organ, or ailment. Moreover, it will help the researcher to make decision in selecting individual or group proteins or genetic materials whose expression needed to be enhanced in EVs for targeted therapy. EVs are very small in nature that have the potential to cross the blood-brain barrier. Hence, beside carrying genetic materials or proteins they could be used as a carrier of bioactive drugs or components in near future. However, despite the high therapeutic potential, any future EV-based therapy must adhere to strict standards with clear safety and risk profile, and a defined mode of action. This could not only lead to a development of affordable cell-free therapeutics but also prevent unproven commercial direct-to-patient exploitation of EV-based therapies that could harm the patients and the credibility of the field.

\section{LIST OF ABBREVIATIONS}

$\begin{array}{ll}\text { Acta2 } & =\text { actin alpha } 2 \\ \text { AD } & =\text { adipose derived } \\ \text { aHSCs } & =\text { activated HSCs } \\ \text { Akt } & =\text { protein kinase B } \\ \text { ALT } & =\text { alanine aminotransferase } \\ \text { AST } & =\text { aspartate aminotransferase } \\ \text { ATP } & =\text { adenosine triphosphate } \\ \text { BM } & =\text { bone marrow } \\ \text { BMSC-EVs } & =\text { BM stem cells derived EVs } \\ \text { CCL } & =\text { C-C motif chemokine ligand } \\ \text { CD } & =\text { cluster of differentiation } \\ \text { CK19 } & =\text { cytokeratin } 19 \\ \text { Colla1 } & =\text { collagen I } \\ \text { CPCs } & =\text { cardiac progenitor cells } \\ \text { CXCR } & =\text { C-X-C motif chemokine receptor }\end{array}$




\begin{tabular}{|c|c|c|}
\hline ECM & $=$ extracellular matrix & Grant, RP158-05/19. The authors would like to thank \\
\hline EPCs & $=$ Endothelial progenitor cells & Umme Salma for reviewing this manuscript. \\
\hline ERK & $=$ extracellular signal-regulated kinase & \\
\hline EVs & $=$ extracellular vesicles & Conflict of Interest \\
\hline GSH & $=$ glutathione & Conflict of Interest \\
\hline GSK-3 & $=$ Glycogen synthase kinase -3 & The authors confirm that this article content has no \\
\hline GSK-3 & $=$ Glycogen synthase kinase -3 & \\
\hline GVHD & $=$ graft versus host disease & Acknowledaments \\
\hline $\mathrm{H} 2 \mathrm{O} 2$ & $=$ hydrogen peroxide & Acknowieagments \\
\hline HGF & $=$ hepatocyte growth factor & \\
\hline HIF & $=$ hypoxia inhibition factor & \\
\hline HLSC & $=$ human adult liver stem cells & \\
\hline $\begin{array}{l}\text { HMGB } 1 \\
\text { HSC }\end{array}$ & $\begin{array}{l}=\text { high mobility group box } 1 \\
=\text { hepatic stellate cells }\end{array}$ & CREDIT AUTHOR CONTRIBUTION STATEMENT \\
\hline HSCs & $=$ hematopoietic stem cells & conceptualization, \\
\hline HSCT & $=$ hematopoietic stem cell therapy & visualization, and writing [original draft, review, and \\
\hline HUVEC & $=$ human umbilical vein endothelial cells & editing] of the manuscript. D.W. and V.G. contributed to \\
\hline hWJ & $=$ human Wharton jelly & the conceptualization, and writing [review, and editing] of \\
\hline IGF-1 & $=$ insulin like growth factor & the manuscript. N.H.A.K. contributed to the \\
\hline IL & $=$ interleukin & conceptualization and writing [original draft, review, and \\
\hline iPSCs & $=$ induced pluripotent stem cells & editing] of the manuscript. L.M.N. contributed to the \\
\hline KGF & $=$ keratinocyte growth factor & writing [review, and editing] of the manuscript. All authors \\
\hline LATS & $=$ large tumor suppressor kinase & approved the final version of the article to be submitted \\
\hline $\mathrm{LDH}$ & $=$ lactate dehydrogenase & and all authors agreed to be accountable for all aspects of \\
\hline MCAo & $=$ middle cerebral artery occlusion & juestions related to \\
\hline MCP-1 & $=$ monocyte chemotactic protein -1 & investigated and resolved. \\
\hline miRNA & $=$ microRNA & \\
\hline
\end{tabular}

MSC-EVs = MSC derived EVs
MSCs = mesenchymal stem cells

$\mathrm{NADH}=$ nicotinamide adenine dinucleotide

hydrogen

$\mathrm{NF}-\kappa \mathrm{B} \quad$ = nuclear factor kappa beta

$\mathrm{NGF} \quad=$ nerve growth factor

$\mathrm{NK} \quad=$ natural killer

p-C-JNK = C-Jun N-terminal kinase

PCNA = proliferating cell nuclear antigen

PI3K = phosphatidylinositol 3-kinase

$\mathrm{Px} \quad=$ peroxidase

qHSCs] = quiescent hepatic stellate cells

SCI $\quad=$ spinal cord injury

SCID = severe combined immunodeficiency

SDF-1 = stromal cell derived factor 1

SHED $\quad=$ stem cells from human

extracted/exfoliated mesenchymal stem cells

$\begin{array}{ll}\text { SOD } & =\text { superoxide dismutase } \\ \text { STAT } & =\text { signal transducer and activator of }\end{array}$

transcription

Tgfbr $\quad=$ TGF $-\beta$ receptor

TGF- $\beta=$ transforming growth factor-beta

Timp = tissue inhibitors of metalloproteinases

TNF = tumor necrosis factor

UC = umbilical cord

UCMSC-EVs = UC MSCs derived EVs

$\mathrm{VEGF} \quad=$ vascular endothelial growth factor

YAP = yes-associated protein

\section{OCRID}

Nazmul Haque: https://orcid.org/0000-0002-8191-8993

Darius Widera: https://orcid.org/0000-0003-1686-130X

Vijayendran Govindasamy: https://orcid.org/0000-00025955-3553

Pratiwi Soesilawati: https://orcid.org/0000-0002-11415645.

Noor Hayaty Abu Kasim: https://orcid.org/0000-00028889-842X

\section{REFERENCES}

1. Collaborators GDaH. Global, regional, and national disability-adjusted life-years [DALYs] for 315 diseases and injuries and healthy life expectancy [HALE], 1990-2015: a systematic analysis for the Global Burden of Disease Study 2015. Lancet. 2016;388[10053]:1603-58.

2. Chen S, Kuhn M, Prettner K, Bloom DE. The macroeconomic burden of noncommunicable diseases in the United States: Estimates and projections. PLOS ONE. 2018;13[11]:e0206702.

3. Mayor S. Non-communicable diseases now cause two thirds of deaths worldwide. BMJ. 2016;355:i5456.

4. Chen X, Pan W. The Treatment Strategies for Neurodegenerative Diseases by Integrative Medicine. Integrative Medicine International. 2014;1[4]:223-5.

5. Said A, Lucey MR. Liver transplantation: an update 2008. Curr Opin Gastroenterol. 2008;24[3]:339-45.

6. Beegle JR, Magner NL, Kalomoiris S, et al. Preclinical evaluation of mesenchymal stem cells overexpressing VEGF to treat critical limb ischemia. Mol Ther Methods Clin Dev. 2016;3:16053.

Consent for Publication

Not applicable.

7. Malliaras K, Marban E. Cardiac cell therapy: where we've been, where we are, and where we should be headed. Br Med Bull. 2011;98:161-85.

8. Beegle J, Lakatos K, Kalomoiris S, et al. Hypoxic preconditioning of mesenchymal stromal cells induces

\section{Funding}


metabolic changes, enhances survival, and promotes cell retention in vivo. Stem Cells. 2015;33[6]:1818-28.

9. Konala VB, Mamidi MK, Bhonde R, Das AK, Pochampally R, Pal R. The current landscape of the mesenchymal stromal cell secretome: A new paradigm for cell-free regeneration. Cytotherapy. 2016;18[1]:13-24.

10. Zakrzewski W, Dobrzyński M, Szymonowicz M, Rybak Z. Stem cells: past, present, and future. Stem Cell Res Ther. 2019;10[1]:68.

11. Geraili A, Jafari P, Hassani MS, et al. Controlling Differentiation of Stem Cells for Developing Personalized Organ-on-Chip Platforms. Advanced Healthcare Materials. 2018;7[2]:1700426.

12. Uz M, Das SR, Ding S, Sakaguchi DS, Claussen JC, Mallapragada SK. Advances in Controlling Differentiation of Adult Stem Cells for Peripheral Nerve Regeneration. Advanced Healthcare Materials. 2018;7[14]:1701046.

13. Santucci L, Bruschi M, Del Zotto G, et al. Biological surface properties in extracellular vesicles and their effect on cargo proteins. Scientific Reports. 2019;9[1]:13048.

14. Rajabzadeh N, Fathi E, Farahzadi R. Stem cellbased regenerative medicine. Stem cell investigation. 2019;6:19-.

15. Shtrichman R, Germanguz I, Eldor JI-. Induced Pluripotent Stem Cells [iPSCs] Derived from Different Cell Sources and their Potential for Regenerative and Personalized Medicine. Curr Mol Med. 2013;13[5]:792805.

16. Haque N, Abdullah BJJ, Kasim NHA. Secretome: Pharmaceuticals for Cell-Free Regenerative Therapy. In: Pham PV, editor. Stem Cell Drugs - A New Generation of Biopharmaceuticals. Stem Cells in Clinical Applications. Cham: Springer International Publishing; 2018. p. 17-35.

17. Stoddart MJ, Bara J, Alini M. Cells and secretome--towards endogenous cell re-activation for cartilage repair. Adv Drug Deliv Rev. 2015;84:135-45.

18. Witman N, Zhou C, Grote Beverborg N, Sahara M, Chien KR. Cardiac progenitors and paracrine mediators in cardiogenesis and heart regeneration. Semin Cell Dev Biol. 2020;100:29-51.

19. Harding C, Heuser J, Stahl P. Endocytosis and intracellular processing of transferrin and colloidal goldtransferrin in rat reticulocytes: demonstration of a pathway for receptor shedding. Eur J Cell Biol. 1984;35[2]:256-63.

20. Pan BT, Johnstone RM. Fate of the transferrin receptor during maturation of sheep reticulocytes in vitro: selective externalization of the receptor. Cell. 1983;33[3]:967-78.

21. Raposo G, Nijman HW, Stoorvogel W, et al. B lymphocytes secrete antigen-presenting vesicles. J Exp Med. 1996;183[3]:1161-72.

22. S ELA, Mager I, Breakefield XO, Wood MJ. Extracellular vesicles: biology and emerging therapeutic opportunities. Nat Rev Drug Discov. 2013;12 [5]:347-57.

23. Raposo G, Stoorvogel W. Extracellular vesicles: exosomes, microvesicles, and friends. J Cell Biol. 2013;200[4]:373-83.

24. Willms E, Johansson HJ, Mager I, et al. Cells release subpopulations of exosomes with distinct molecular and biological properties. Sci Rep. 2016;6:22519.
25. Cocucci E, Racchetti G, Meldolesi J. Shedding microvesicles: artefacts no more. Trends Cell Biol. 2009;19[2]:43-51.

26. Atkin-Smith GK, Tixeira R, Paone S, et al. A novel mechanism of generating extracellular vesicles during apoptosis via a beads-on-a-string membrane structure. Nat Commun. 2015;6:7439.

27. Bakhshandeh B, Kamaleddin M, Aalishah K. A Comprehensive Review on Exosomes and Microvesicles as Epigenetic Factors. Current Stem Cell Research \& Therapy. 2016;12[1]:31-6.

28. Matula Z, Nemeth A, Lorincz P, et al. The Role of Extracellular Vesicle and Tunneling NanotubeMediated Intercellular Cross-Talk Between Mesenchymal Stem Cells and Human Peripheral T Cells. Stem Cells Dev. 2016;25[23]:1818-32.

29. Alcayaga-Miranda F, Varas-Godoy M, Khoury M. Harnessing the Angiogenic Potential of Stem CellDerived Exosomes for Vascular Regeneration. Stem Cells Int. 2016;2016:3409169.

30. Mrvar-Brecko A, Sustar V, Jansa V, et al. Isolated microvesicles from peripheral blood and body fluids as observed by scanning electron microscope. Blood Cells Mol Dis. 2010;44[4]:307-12.

31. Ratajczak J, Miekus K, Kucia M, et al. Embryonic stem cell-derived microvesicles reprogram hematopoietic progenitors: evidence for horizontal transfer of mRNA and protein delivery. Leukemia. 2006;20[5]:847-56.

32. Quesenberry PJ, Aliotta JM. Cellular phenotype switching and microvesicles. Adv Drug Deliv Rev. 2010;62[12]:1141-8.

33. Rosca AM, Rayia DM, Tutuianu R. Emerging Role of Stem Cells - Derived Exosomes as Valuable Tools for Cardiovascular Therapy. Curr Stem Cell Res Ther. 2017;12[2]:134-8.

34. Cervio E, Barile L, Moccetti T, Vassalli G. Exosomes for Intramyocardial Intercellular Communication. Stem Cells Int. 2015;2015:482171.

35. Sun K, Zhou Z, Ju X, et al. Combined transplantation of mesenchymal stem cells and endothelial progenitor cells for tissue engineering: a systematic review and meta-analysis. Stem Cell Res Ther. 2016;7[1]:151.

36. Zhang H, Xiang M, Meng D, Sun N, Chen S. Inhibition of Myocardial Ischemia/Reperfusion Injury by Exosomes Secreted from Mesenchymal Stem Cells. Stem Cells Int. 2016;2016:4328362.

37. Lai RC, Arslan F, Lee MM, et al. Exosome secreted by MSC reduces myocardial ischemia/reperfusion injury. Stem Cell Res. 2010;4[3]:214-22.

38. Bobis-Wozowicz S, Kmiotek K, Sekula M, et al. Human Induced Pluripotent Stem Cell-Derived Microvesicles Transmit RNAs and Proteins to Recipient Mature Heart Cells Modulating Cell Fate and Behavior. Stem Cells. 2015;33[9]:2748-61.

39. Lai RC, Yeo RW, Tan KH, Lim SK. Mesenchymal stem cell exosome ameliorates reperfusion injury through proteomic complementation. Regen Med. 2013;8[2]:197-209.

40. Arslan F, Lai RC, Smeets MB, et al. Mesenchymal stem cell-derived exosomes increase ATP levels, decrease oxidative stress and activate PI3K/Akt pathway to enhance myocardial viability and prevent 
adverse remodeling after myocardial ischemia/reperfusion injury. Stem Cell Res. 2013;10[3]:301-12.

41. Ibrahim AG, Cheng K, Marban E. Exosomes as critical agents of cardiac regeneration triggered by cell therapy. Stem Cell Reports. 2014;2[5]:606-19.

42. Zernecke A, Bidzhekov K, Noels $\mathrm{H}$, et al. Delivery of microRNA-126 by apoptotic bodies induces CXCL12-dependent vascular protection. Sci Signal. 2009;2[100]:ra81.

43. Ong SG, Lee WH, Huang M, et al. Cross talk of combined gene and cell therapy in ischemic heart disease: role of exosomal microRNA transfer. Circulation. 2014;130[11 Suppl 1]:S60-9.

44. Barile L, Lionetti V, Cervio E, et al. Extracellular vesicles from human cardiac progenitor cells inhibit cardiomyocyte apoptosis and improve cardiac function after myocardial infarction. Cardiovasc Res. 2014;103[4]:530-41.

45. Dai B, Pan Q, Li Z, et al. Multilayer Membranes of Glycosaminoglycans and Collagen I Biomaterials Modulate the Function and Microvesicle Release of Endothelial Progenitor Cells. Stem Cells Int. 2016;2016:4796578.

46. Chen T, Bai H, Shao Y, et al. Stromal cellderived factor-1/CXCR4 signaling modifies the capillarylike organization of human embryonic stem cell-derived endothelium in vitro. Stem Cells. 2007;25[2]:392-401.

47. Kang K, Ma R, Cai W, et al. Exosomes Secreted from CXCR4 Overexpressing Mesenchymal Stem Cells Promote Cardioprotection via Akt Signaling Pathway following Myocardial Infarction. Stem Cells Int. 2015;2015:659890.

48. Hernandez-Gea V, Friedman SL. Pathogenesis of liver fibrosis. Annu Rev Pathol. 2011;6:425-56.

49. Fonsato V, Collino F, Herrera MB, et al. Human liver stem cell-derived microvesicles inhibit hepatoma growth in SCID mice by delivering antitumor microRNAs. Stem Cells. 2012;30[9]:1985-98.

50. Bruno S, Collino F, Deregibus MC, Grange C, Tetta C, Camussi G. Microvesicles derived from human bone marrow mesenchymal stem cells inhibit tumor growth. Stem Cells Dev. 2013;22[5]:758-71.

51. Nong K, Wang W, Niu X, et al. Hepatoprotective effect of exosomes from human-induced pluripotent stem cell-derived mesenchymal stromal cells against hepatic ischemia-reperfusion injury in rats. Cytotherapy. 2016;18[12]:1548-59.

52. Huang R, Pan Q, Ma X, et al. Hepatic Stellate Cell-Derived Microvesicles Prevent Hepatocytes from Injury Induced by APAP/H2O2. Stem Cells Int. 2016;2016:8357567.

53. Li T, Yan Y, Wang B, et al. Exosomes derived from human umbilical cord mesenchymal stem cells alleviate liver fibrosis. Stem Cells Dev. 2013;22[6]:84554.

54. Tan CY, Lai RC, Wong W, Dan YY, Lim SK, Ho HK. Mesenchymal stem cell-derived exosomes promote hepatic regeneration in drug-induced liver injury models. Stem Cell Res Ther. 2014;5[3]:76.

55. Higashi T, Friedman SL, Hoshida Y. Hepatic stellate cells as key target in liver fibrosis. Adv Drug Deliv Rev. 2017;121:27-42.
56. Tsuchida T, Friedman SL. Mechanisms of hepatic stellate cell activation. Nat Rev Gastroenterol Hepatol. 2017;14[7]:397-411.

57. Fierabracci A, Del Fattore A, Luciano R, Muraca M, Teti A, Muraca M. Recent advances in mesenchymal stem cell immunomodulation: the role of microvesicles. Cell Transplant. 2015;24[2]:133-49.

58. Kisseleva T, Cong M, Paik $\mathrm{Y}$, et al. Myofibroblasts revert to an inactive phenotype during regression of liver fibrosis. Proc Natl Acad Sci U S A. 2012;109[24]:9448-53.

59. Li L, Piontek K, Ishida M, et al. Extracellular vesicles carry microRNA-195 to intrahepatic cholangiocarcinoma and improve survival in a rat model. Hepatology. 2017;65[2]:501-14.

60. Luarte A, Batiz LF, Wyneken U, Lafourcade C. Potential Therapies by Stem Cell-Derived Exosomes in CNS Diseases: Focusing on the Neurogenic Niche. Stem Cells Int. 2016;2016:5736059.

61. Farinazzo A, Turano E, Marconi S, Bistaffa E, Bazzoli E, Bonetti B. Murine adipose-derived mesenchymal stromal cell vesicles: in vitro clues for neuroprotective and neuroregenerative approaches. Cytotherapy. 2015;17[5]:571-8.

62. Jarmalaviciute A, Tunaitis V, Pivoraite U, Venalis A, Pivoriunas A. Exosomes from dental pulp stem cells rescue human dopaminergic neurons from 6-hydroxydopamine-induced apoptosis. Cytotherapy. 2015;17[7]:932-9.

63. Xin H, Li Y, Buller B, et al. Exosome-mediated transfer of miR-133b from multipotent mesenchymal stromal cells to neural cells contributes to neurite outgrowth. Stem Cells. 2012;30[7]:1556-64.

64. Xin H, Li Y, Liu Z, et al. MiR-133b promotes neural plasticity and functional recovery after treatment of stroke with multipotent mesenchymal stromal cells in rats via transfer of exosome-enriched extracellular particles. Stem Cells. 2013;31[12]:2737-46.

65. Rong Y, Liu W, Wang J, et al. Neural stem cellderived small extracellular vesicles attenuate apoptosis and neuroinflammation after traumatic spinal cord injury by activating autophagy. Cell Death Dis. 2019;10[5]:340.

66. Lu Y, Zhou Y, Zhang R, et al. Bone Mesenchymal Stem Cell-Derived Extracellular Vesicles Promote Recovery Following Spinal Cord Injury via Improvement of the Integrity of the Blood-Spinal Cord Barrier. Frontiers in neuroscience. 2019;13:209-.

67. Romanelli P, Bieler L, Scharler C, et al. Extracellular Vesicles Can Deliver Anti-inflammatory and Anti-scarring Activities of Mesenchymal Stromal Cells After Spinal Cord Injury. Frontiers in Neurology. 2019;10[1225].

68. Yu T, Zhao C, Hou S, Zhou W, Wang B, Chen Y. Exosomes secreted from miRNA-29b-modified mesenchymal stem cells repaired spinal cord injury in rats. Braz J Med Biol Res. 2019;52[12]:e8735.

69. Vikartovska Z, Kuricova M, Farbakova J, et al. Stem Cell Conditioned Medium Treatment for Canine Spinal Cord Injury: Pilot Feasibility Study. International journal of molecular sciences. 2020;21[14]:5129.

70. Chen CC, Liu L, Ma F, et al. Elucidation of Exosome Migration across the Blood-Brain Barrier Model In Vitro. Cell Mol Bioeng. 2016;9[4]:509-29. 
71. Tonnesen MG, Feng X, Clark RA. Angiogenesis in wound healing. J Investig Dermatol Symp Proc. 2000;5[1]:40-6.

72. Shabbir A, Cox A, Rodriguez-Menocal L, Salgado M, Van Badiavas E. Mesenchymal Stem Cell Exosomes Induce Proliferation and Migration of Normal and Chronic Wound Fibroblasts, and Enhance Angiogenesis In Vitro. Stem Cells Dev. 2015;24[14]:163547.

73. Deregibus MC, Cantaluppi V, Calogero R, et al. Endothelial progenitor cell derived microvesicles activate an angiogenic program in endothelial cells by a horizontal transfer of mRNA. Blood. 2007;110[7]:2440-8.

74. Nagano $M$, Yamashita $T$, Hamada $H$, et al. Identification of functional endothelial progenitor cells suitable for the treatment of ischemic tissue using human umbilical cord blood. Blood. 2007;110[1]:151-60.

75. Tu TC, Nagano M, Yamashita $T$, et al. A Chemokine Receptor, CXCR4, Which Is Regulated by Hypoxia-Inducible Factor 2alpha, Is Crucial for Functional Endothelial Progenitor Cells Migration to Ischemic Tissue and Wound Repair. Stem Cells Dev. 2016;25[3]:266-76.

76. Fang S, Xu C, Zhang Y, et al. Umbilical CordDerived Mesenchymal Stem Cell-Derived Exosomal MicroRNAs Suppress Myofibroblast Differentiation by Inhibiting the Transforming Growth Factor-beta/SMAD2 Pathway During Wound Healing. Stem Cells Transl Med. 2016;5[10]:1425-39.

77. Zhang B, Wang M, Gong A, et al. HucMSCExosome Mediated-Wnt4 Signaling Is Required for Cutaneous Wound Healing. Stem Cells. 2015;33[7]:215868.

78. Zhang B, Shi Y, Gong A, et al. HucMSC Exosome-Delivered 14-3-3zeta Orchestrates Self-Control of the Wnt Response via Modulation of YAP During Cutaneous Regeneration. Stem Cells. 2016;34[10]:2485500 .

79. Nair R, Santos L, Awasthi S, et al. Extracellular vesicles derived from preosteoblasts influence embryonic stem cell differentiation. Stem Cells Dev. 2014;23[14]:1625-35.

80. Martins M, Ribeiro D, Martins A, Reis RL, Neves NM. Extracellular Vesicles Derived from Osteogenically Induced Human Bone Marrow Mesenchymal Stem Cells Can Modulate Lineage Commitment. Stem Cell Reports. 2016;6[3]:284-91.

81. Narayanan R, Huang CC, Ravindran S. Hijacking the Cellular Mail: Exosome Mediated Differentiation of Mesenchymal Stem Cells. Stem Cells Int. 2016;2016:3808674.

82. Furuta T, Miyaki S, Ishitobi $\mathrm{H}$, et al. Mesenchymal Stem Cell-Derived Exosomes Promote Fracture Healing in a Mouse Model. Stem Cells Transl Med. 2016;5[12]:1620-30.

83. Berglund AK, Fortier LA, Antczak DF, Schnabel LV. Immunoprivileged no more: measuring the immunogenicity of allogeneic adult mesenchymal stem cells. Stem cell research \& therapy. 2017;8[1]:288-.

84. Ankrum JA, Ong JF, Karp JM. Mesenchymal stem cells: immune evasive, not immune privileged. Nat Biotechnol. 2014;32[3]:252-60.

85. Zhao L, Chen S, Yang P, Cao H, Li L. The role of mesenchymal stem cells in hematopoietic stem cell transplantation: prevention and treatment of graft-versus- host disease. Stem Cell Research \& Therapy. 2019;10[1]:182.

86. Elgaz S, Kuçi Z, Kuçi S, Bönig H, Bader P. Clinical Use of Mesenchymal Stromal Cells in the Treatment of Acute Graft-versus-Host Disease. Transfusion Medicine and Hemotherapy. 2019;46[1]:2734.

87. Giebel B, Kordelas L, Börger V. Clinical potential of mesenchymal stem/stromal cell-derived extracellular vesicles. Stem cell investigation. 2017;4:84-.

88. Gomzikova MO, James V, Rizvanov AA. Therapeutic Application of Mesenchymal Stem Cells Derived Extracellular Vesicles for Immunomodulation. Frontiers in Immunology. 2019;10[2663].

89. Xie M, Xiong W, She Z, et al. Immunoregulatory Effects of Stem Cell-Derived Extracellular Vesicles on Immune Cells. Frontiers in Immunology. 2020;11[13].

90. Seo Y, Kim H-S, Hong I-S. Stem Cell-Derived Extracellular Vesicles as Immunomodulatory Therapeutics. Stem cells international. 2019;2019:5126156-.

91. Wang L, Gu Z, Zhao X, et al. Extracellular Vesicles Released from Human Umbilical Cord-Derived Mesenchymal Stromal Cells Prevent Life-Threatening Acute Graft-Versus-Host Disease in a Mouse Model of Allogeneic Hematopoietic Stem Cell Transplantation. Stem Cells Dev. 2016;25[24]:1874-83.

92. Del Fattore A, Luciano R, Pascucci L, et al. Immunoregulatory Effects of Mesenchymal Stem CellDerived Extracellular Vesicles on T Lymphocytes. Cell Transplant. 2015;24[12]:2615-27.

93. Kordelas L, Rebmann V, Ludwig AK, et al. MSC-derived exosomes: a novel tool to treat therapyrefractory graft-versus-host disease. Leukemia. 2014;28[4]:970-3.

94. Di Trapani M, Bassi G, Midolo M, et al. Differential and transferable modulatory effects of mesenchymal stromal cell-derived extracellular vesicles on T, B and NK cell functions. Sci Rep. 2016;6:24120.

95. Koch M, Lemke A, Lange C. Extracellular Vesicles from MSC Modulate the Immune Response to Renal Allografts in a MHC Disparate Rat Model. Stem Cells Int. 2015;2015:486141.

96. Kordelas L, Schwich E, Dittrich R, et al. Individual immune-modulatory capabilities of MSCderived extracellular vesicle [EV] preparations and recipient-dependent responsiveness. International Journal of Molecular Sciences. 2019;20[7].

97. Qi S, Wu D. Bone marrow-derived mesenchymal stem cells protect against cisplatin-induced acute kidney injury in rats by inhibiting cell apoptosis. Int $\mathbf{J}$ Mol Med. 2013;32[6]:1262-72.

98. Duffield JS, Park KM, Hsiao LL, et al. Restoration of tubular epithelial cells during repair of the postischemic kidney occurs independently of bone marrow-derived stem cells. J Clin Invest. 2005;115[7]:1743-55.

99. Peired AJ, Sisti A, Romagnani P. Mesenchymal Stem Cell-Based Therapy for Kidney Disease: A Review of Clinical Evidence. Stem Cells Int. 2016;2016:4798639.

100. Tomasoni S, Longaretti L, Rota C, et al. Transfer of growth factor receptor mRNA via exosomes unravels the regenerative effect of mesenchymal stem cells. Stem Cells Dev. 2013;22[5]:772-80. 
101. Gu D, Zou X, Ju G, Zhang G, Bao E, Zhu Y. Mesenchymal Stromal Cells Derived Extracellular Vesicles Ameliorate Acute Renal Ischemia Reperfusion Injury by Inhibition of Mitochondrial Fission through miR-30. Stem Cells Int. 2016;2016:2093940.

102. Akcay A, Nguyen Q, Edelstein CL. Mediators of inflammation in acute kidney injury. Mediators Inflamm. 2009;2009:137072.

103. Du T, Zhu YJ. The regulation of inflammatory mediators in acute kidney injury via exogenous mesenchymal stem cells. Mediators Inflamm. 2014;2014:261697.

104. Shen B, Liu J, Zhang F, et al. CCR2 Positive Exosome Released by Mesenchymal Stem Cells Suppresses Macrophage Functions and Alleviates Ischemia/Reperfusion-Induced Renal Injury. Stem Cells Int. 2016;2016:1240301.

105. Maffi P, Secchi A. Clinical results of islet transplantation. Pharmacol Res. 2015;98:86-91.

106. Cantaluppi V, Biancone L, Figliolini F, et al. Microvesicles derived from endothelial progenitor cells enhance neoangiogenesis of human pancreatic islets. Cell Transplant. 2012;21[6]:1305-20.

107. Aliotta JM, Sanchez-Guijo FM, Dooner GJ, et al. Alteration of marrow cell gene expression, protein production, and engraftment into lung by lung-derived microvesicles: a novel mechanism for phenotype modulation. Stem Cells. 2007;25[9]:2245-56.

108. Aliotta JM, Lee D, Puente N, et al. Progenitor/stem cell fate determination: interactive dynamics of cell cycle and microvesicles. Stem Cells Dev. 2012;21[10]:1627-38.

109. Aliotta JM, Pereira M, Wen S, et al. Exosomes induce and reverse monocrotaline-induced pulmonary hypertension in mice. Cardiovascular Research. 2016;110[3]:319-30.

110. Zhu YG, Feng XM, Abbott J, et al. Human mesenchymal stem cell microvesicles for treatment of Escherichia coli endotoxin-induced acute lung injury in mice. Stem Cells. 2014;32[1]:116-25.

111. Di Rocco G, Baldari S, Toietta G. Towards Therapeutic Delivery of Extracellular Vesicles: Strategies for In Vivo Tracking and Biodistribution Analysis. Stem Cells Int. 2016;2016:5029619.

112. Kubikova I, Konecna H, Sedo O, et al. Proteomic profiling of human embryonic stem cell-derived microvesicles reveals a risk of transfer of proteins of bovine and mouse origin. Cytotherapy. 2009;11[3]:330-40, $1 \mathrm{p}$ following 40 . 